\title{
Targeting of Prosurvival Pathways as Therapeutic Approaches against Primary Effusion Lymphomas: Past, Present, and Future
}

\author{
Marisa Granato, Roberta Santarelli, Roberta Gonnella, Antonella Farina, \\ Pankaj Trivedi, Alberto Faggioni, and Mara Cirone \\ Department of Experimental Medicine, Istituto Pasteur Fondazione Cenci Bolognetti, Sapienza University, 00161 Rome, Italy \\ Correspondence should be addressed to Alberto Faggioni; alberto.faggioni@uniroma1.it \\ and Mara Cirone; mara.cirone@uniromal.it
}

Received 28 February 2014; Accepted 29 October 2014

Academic Editor: Panayiotis Panayiotidis

Copyright (C) 2015 Marisa Granato et al. This is an open access article distributed under the Creative Commons Attribution License, which permits unrestricted use, distribution, and reproduction in any medium, provided the original work is properly cited.

Constitutively activated prosurvival pathways render cancer cells addicted to their effects. Consequently they turn out to be the Achilles' heels whose inhibition can be exploited in anticancer therapy. Primary effusion lymphomas (PELs) are very aggressive non-Hodgkin's B cell lymphomas, whose pathogenesis is strictly linked to Kaposi's sarcoma herpesvirus (KSHV) infection. Here we summarized previous studies from our and other laboratories exploring the cytotoxic effect of drugs inhibiting the main prosurvival pathways activated in PEL cells. Moreover, the immunogenicity of cell death, in terms of dendritic cell (DC) activation and their potential side effect on DCs, is discussed.

\section{Primary Effusion Lymphomas' Biology}

Primary effusion lymphomas (PELs) are very aggressive nonHodgkin's B cell lymphomas with poor prognosis that frequently arise in immune-compromised patients [1]. PELs originate from postgerminal center B cells and exhibit indeterminate immune-phenotypes; that is, they express CD45, CD138, and activation-associated antigens (CD30, CD38, and HLA-DR) and lack surface expression of $B$ cell markers (CD19, CD20, CD79a, and immunoglobulin) but exhibit clonal rearrangements and somatic hypermutation of the immunoglobulin genes. PELs are strictly associated with a DNA oncovirus, the Kaposi's sarcoma herpesvirus (KSHV), and, in the majority of cases, they are dually infected by a second oncovirus belonging to the gamma-herpesvirus family $[2,3]$, the Epstein-Barr virus (EBV) [4]. The low response to conventional therapies indicates that new therapeutic strategies are needed for PELs. PEL tumor growth relays on the presence of cytokines that, produced by the tumor itself [5], stimulate their own release with an autocrine positive feedback loop. An important role in PEL survival has been reported for interleukin- (IL-) 6, IL-10, vascular endothelial growth factor (VEGF), and Oncostatin M [6-8]. In addition to cellular cytokines, KSHV encodes its own viral cytokines such as IL-6 which is similar to human IL- 6 and exerts an important prosurvival effect on PEL cells $[6,9]$ and macrophage inhibitory proteins (MIPs) that affect the immune system of the infected host [10].

\section{Prosurvival Molecules in PELs}

PEL cell survival relays on the constitutive activation of several pathways and also on the hyperexpression of several heat shock proteins (HSPs), a characteristic that is common among the cancer cells.

2.1. JAK2/STAT3 Pathway. By engagement of their specific cellular receptors, cytokines such as IL-6 and IL-10 and VEGF activate the JAK2/STAT3 transduction pathway that promotes the transcription of genes encoding for proteins involved in PEL resistance to apoptosis and contributes to tumor survival [11-13]. Consequently, the specific inhibition of STAT3 activation by AG490 has been reported to induce PEL cell death by downregulating survivin expression [14]. In addition, we have recently reported that AG490 is able to trigger an immunogenic apoptosis in PEL cells [15]. This aspect is of pivotal importance since it is now clear enough that, 
without the contribution of the immune system, obtained by triggering an immunogenic cell death type and/or the reversion of the tumor induced immunosuppression [16], no complete eradication of cancer can be achieved by anticancer therapies $[17,18]$. Moreover AG490 is able to revert immunosuppression caused by tumor-released factors or induced by tumor viruses such as KSHV, which correlates with STAT3 activation in immune cells $[19,20]$. This immune-stimulating AG490 property further encourages its use in PEL anticancer therapy.

2.2. PI3K/AKT/mTOR Pathway. It is known that, in addition to STAT3, cytokines like IL-6 and IL-10, VEGF, and Oncostatin M [21] are able to activate other pathways such as $\mathrm{PI} 3 \mathrm{~K} / \mathrm{AKT}$ and Ras/MAPK that govern fundamental processes [22], such as cell proliferation, differentiation, metabolism, and tumor survival. The PI3K/AKT is constitutively activated in PELs due to the effect mediated by PEL released cytokines and following KSHV infection [23], in particular as an effect of K1 viral protein expression [24]. Previous papers have reported that targeting PI3K/AKT pathway in PEL cells may represent an effective anticancer strategy [25, 26]. We have reported that KSHV infection hyperactivates AKT in THP-1 cells and, as a consequence, the response to proteasome inhibition is reduced. Moreover, AKT activation also leads to Glutl membrane localization, which is known to promote cell survival by increasing the glucose uptake. On the other hand, we found that Glutl translocation renders THP1 cells more susceptible to the glycolysis inhibitor 2-deoxyD-glucose (DG) effect [27]. This is another example of how a prosurvival effect turns out to be a prodeath effect in tumor cells. Finally, since PI3K/AKT activation leads to the phosphorylation of the downstream molecule mTOR, also its inhibition has been exploited in PEL therapy [28]. mTOR inhibition has been shown to reduce PEL cell survival by interfering with the release of cytokines known to be essential for PEL cell growth, and more recently it has been shown that mTOR inhibition can be effective also against a second KSHV-associated malignancy such as Kaposi's sarcoma.

2.3. MAPK Pathways. Mitogen activated protein kinases (MAPKs) including ERKs, JNKs, and p38 kinases control a vast array of physiological processes [29]. They can be activated in tumor cells by several stimuli such as stress conditions and inflammatory cytokines, also produced by tumor itself. Their inhibition can be potentially explorable in PEL therapy, considering that a cross-talk between these pathways and other prosurvival pathways, such as NF- $\kappa \mathrm{B}$ and $\mathrm{PI} 3 \mathrm{~K} / \mathrm{AKT} / \mathrm{m}-\mathrm{TOR}$, has been reported [30]. In a recent study, we have shown that JNK inhibition enhances Bortezomibinduced cell death and that SP600125 JNK inhibitor is also able to induce PEL cell death to some extent [31]. A prosurvival role of JNK2 activation during the ER stress caused by tunicamycin treatment has also been shown in a different cell type [32]. In addition, p38 MAPK seems to be activated in PEL cells, in particular in those harbouring KSHV, in comparison with KSHV-negative PEL cells [4].
2.4. NF- $\kappa B$ Pathway. An essential role in PEL cell survival is known to be played by NF- $\kappa \mathrm{B}$, constitutively activated in PEL cells due also to the effect mediated by KSHV-encoded viral FLICE-inhibitory protein (v-FLIP) expression [33, 34]. It has been reported that NF- $\kappa$ B inhibition with Bayl1-7082 exerts a strong reduction of PEL cell survival [33]. Moreover, the Bortezomib cytotoxic effect, previously observed on PEL cells, also involves NF- $\kappa \mathrm{B}$ inhibition [35], even if its main cytotoxic effect is proteasome inhibition [36]. We have previously explored the immunogenicity of Bortezomib-induced cell death in PEL and showed that Bortezomib was able to induce damage associated molecular patterns (DAMPs) expression on the surface of apoptotic PEL cells, which then resulted in dendritic cell (DC) activation [15, 37]. Moreover, we have demonstrated that Bortezomib induces endoplasmic reticulum (ER) stress and a prosurvival autophagy in PEL cells, due to the accumulation of ubiquitinated proteins consequent to the inhibition of proteasomal degradation. The autophagic blockage, during Bortezomib treatment, further increased its cytotoxic effect. Thus this strategy could be explored in the cancer therapy against PELs [31].

2.5. HSPs. A further effect mediated by the activation of pathways such as Ras/MAPK and JAK/STAT3 is the increase of heat shock protein (HSP) expression [38, 39]. HSPs are known to help cancer cells to survive in the stressful conditions caused by their rapid growth and nutrient shortage. HSPs also counteract the cytotoxic effects induced by chemotherapeutic treatments. For this reason they represent a target for anticancer therapy [38], also considering that their expression, and consequently the cytotoxic effect of their inhibition, is low in normal cells compared with cancer cells. Accordingly, we have recently reported that the inhibition of HSP70, using the small molecule 2-phenylethynesulfonamide (PES), is a successful therapeutic strategy against PEL cells and that PES showed very low cytotoxic effect on normal B cells from which PEL cells arise [40]. PES induced lysosome permeabilization and a necroptotic cell death type in PEL cells with immunogenic properties toward DCs. It represents a valid strategy against this cancer and possibly against other tumors that, displaying oncosuppressor mutations, may be resistant to apoptosis inducing drugs. Also the inhibition of HSP90, another chaperone protein with the essential function in protein correct folding, has been reported to be effective against PELs $[41,42]$, as well as against multiple myeloma, which shares many similarities with PELs $[43,44]$.

\section{Antiviral Strategies in PELs}

PEL cells harbour KSHV, which persists in a latent state in the majority of the cells. Upon appropriate stimuli, latent infection can be switched into lytic productive infection that generally leads to cell lysis and spread of the viral particles. The possibility to kill tumor cells by inducing viral replication can be explored in the therapy against tumors latently infected with DNA viruses, especially because during viral replication tumor cells become sensitive to the effects induced by the 


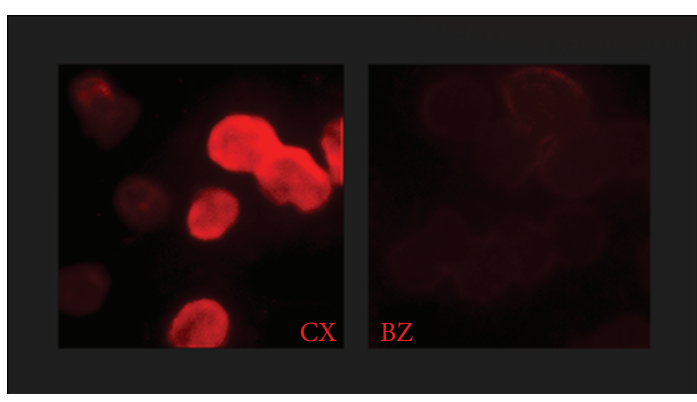

(a)

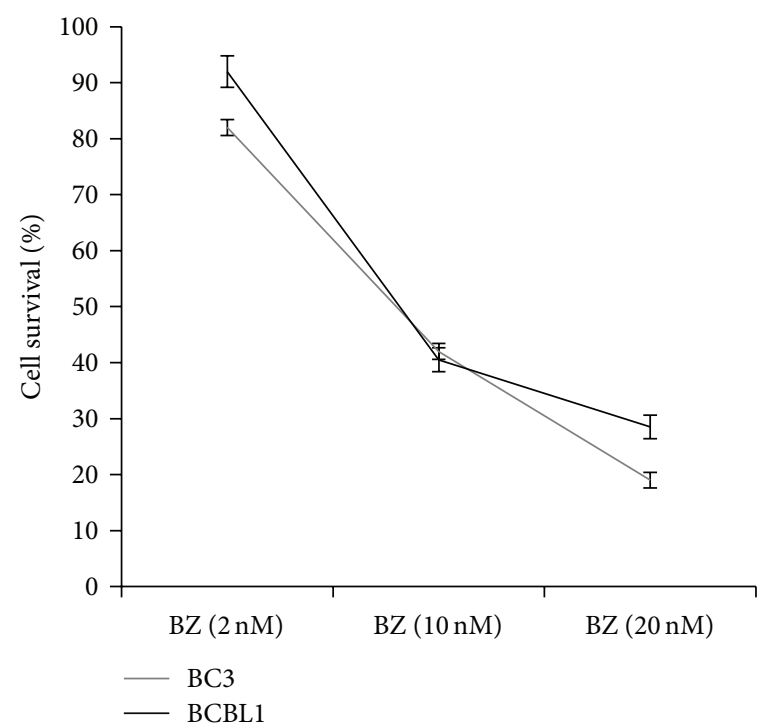

(b)

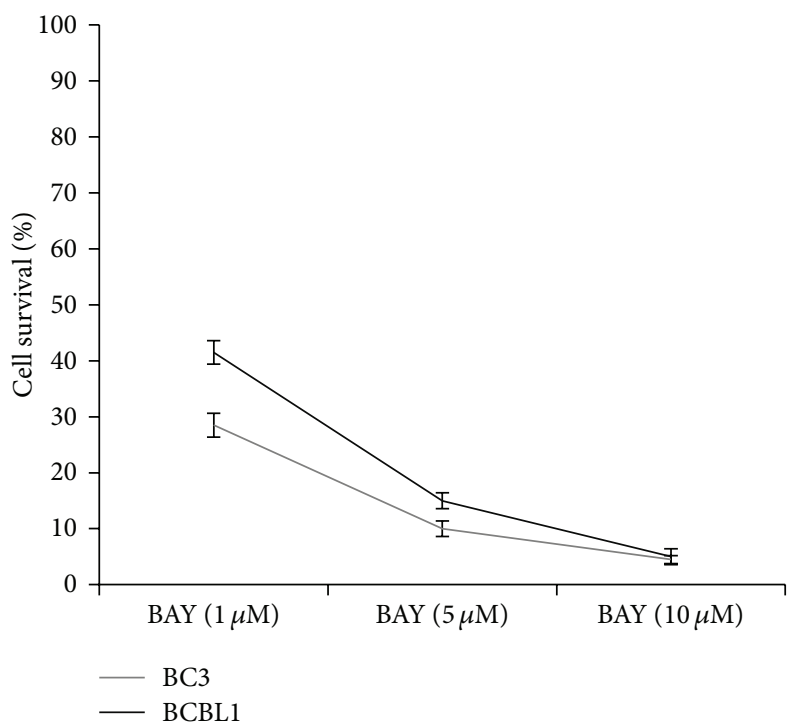

(c)

Figure 1: Effect of Bortezomib (BZ) (Santa Cruz) or Bayl1-7082 (BAY) (Santa Cruz) on BC3 and BCBL1 PEL cells. (a) Effect on NF- $\kappa$ B p65 nuclear localization of Bortezomib in BC3 cells, in comparison to the untreated control cells, analyzed by immunofluorescence assay (IFA). A representative experiment out of three is shown. (b and c) Trypan blue exclusion assays of BC3 and BCBL1 PEL cells treated for 24 hours with Bortezomib (BZ) or Bayll-7082 (BAY), at the indicated doses. The mean \pm SD of three independent experiments is reported.

antiviral drugs. For the above reasons, strategies that allow the manipulation of viral life cycle, reducing or promoting viral production, are potentially explorable in PELs. One of the strategies that allow manipulating the viral life cycle is the modulation of the cellular autophagy which has been shown to have a strong impact on KSHV replication [45].

\section{Experimental Data}

Here we have summarized our and other experimental evidences showing the cytotoxic effect mediated by the pharmacological inhibition of all these pathways in PEL cell lines.
We first confirmed that indirectly inhibiting NF- $\kappa \mathrm{B}$ with Bortezomib (Figure 1(a)) a dose-dependent reduction of PEL cell survival can be observed (Figure 1(b)), confirming our previous reported results [15]. Even stronger cytotoxic activity on PEL cells was obtained with Bayl1-7082 NF- $\kappa$ B, specific inhibitor [46], that was indeed able to kill the majority of PEL cells at the concentration of $5 \mathrm{mM}$ (Figure 1(c)). Next, given the importance of JAK2/STAT3 activation in PEL survival and in the reversion of immunosuppression, reported by us as well as by other groups $[14,15,47]$, we performed a doseresponse assay pharmacologically inhibiting STAT3 activation with AG490 and confirmed that it was very effective in reducing PEL cell survival. Besides its efficacy against PEL 


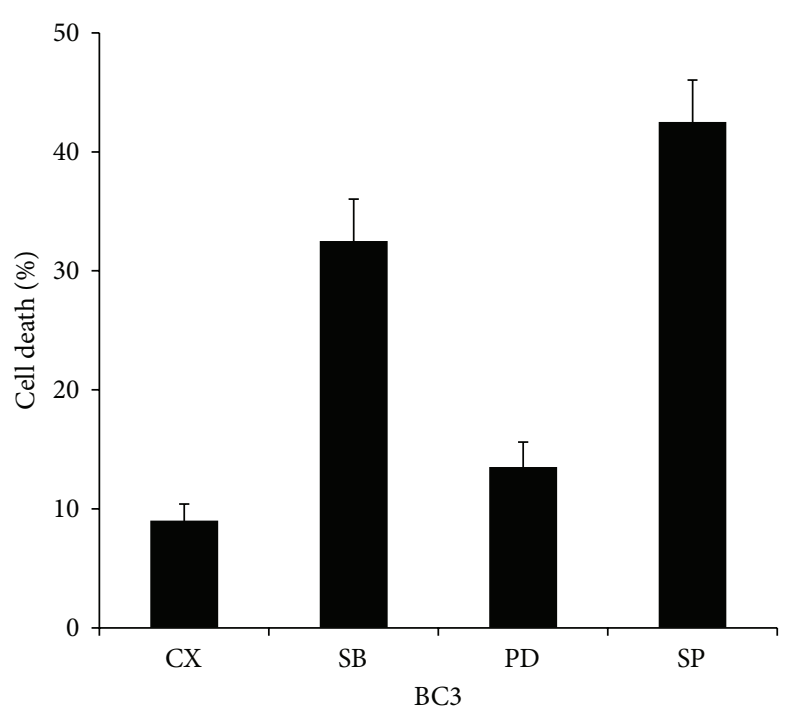

(a)

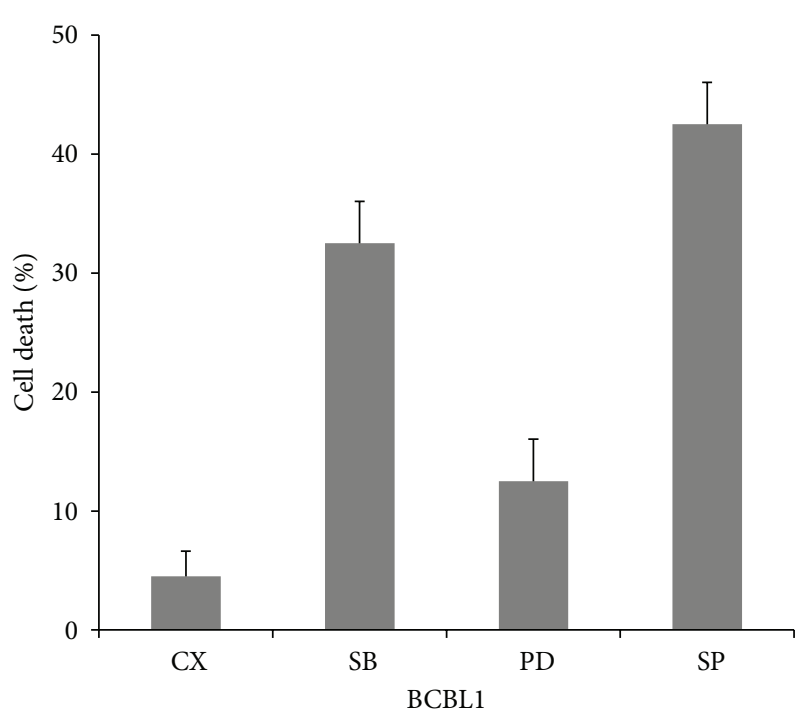

(b)

FIGURE 2: Effect on PEL cell cytotoxicity of p38, JNK, or ERK inhibition by SB203580, SP600125, and PD98059, respectively, based on trypan blue exclusion is reported. The treatment was performed for 24 hours at the concentration of $10 \mu \mathrm{M}$. All the reagents were purchased from Santa Cruz. The mean \pm SD of three independent experiments is reported.

cells, the use of AG490 is also encouraged by our previous observations showing that it can induce an immunogenic cell death in these cells and has a low side effect on dendritic cell viability $[15,38]$. Next, a dose-response treatment aimed at the targeting of PI3K/AKT pathway was then performed with AKT inhibitor LY294002 against BC3 and BCBL1 PEL cells. LY294002, used at the concentration of $20 \mathrm{mM}$, induced about 50\% reduction of PEL cell survival, after 24 hours of treatment (Figure 2). One of the consequences of AKT activation is a change in the cell metabolism, such as an increase of cell resistance to glycolysis inhibitors. Thus, it will be interesting to investigate how treatment LY294002 would affect PEL cell resistance to glucose starvation. A low cytotoxic effect on PEL cells was observed with rapamycin mTOR inhibitor, used at $50 \mathrm{nM}$, for 24 hours. However, its combination with autophagy inhibitor 3-methyladenine (3$\mathrm{MA}$ ) resulted in a higher cytotoxic effect (unpublished data). This is in agreement with the notion that inhibition of mTOR induces autophagy that usually helps cells to survive during starvation or stressful conditions; thus its inhibition may increase cell death.

Additionally, the potential cytotoxic effect of the inhibition of JNK, p38, and ERK MAPK pathways was evaluated on PEL cells. The results shown in Figure 3 indicate that the higher cytotoxic effect was obtained by using SP600125 JNK inhibitor, confirming that this pathway plays an important prosurvival role in PEL cells, according to previously reported studies [31]. All MAPK inhibitors were used at the concentration of $10 \mathrm{mM}$ and a low cytotoxicity was also observed with the inhibitor of p38 MAPK SB203580 and with the ERK inhibitor PD98059, according to a recent study [48] (Figure 3).

The activation of the above-mentioned prosurvival pathway leads to upregulation of HSPs, which are classified based on their molecular weight. They play multiple roles in cancer cells; for example, they ensure the correct protein folding, which is very important especially for cancer cells. Moreover, HSPs are required for the expression of some KSHV essential proteins $[49,50]$. The major role in cancer cell survival is played by HSP70 and HSP90. The cytotoxicity of HSP70 inhibitor 2-phenylethynesulfonamide (PES) against PEL cells was previously reported by our group [40]. Here we compared the effect of PES with Benzisoxazole HSP90 inhibitor, considering that HSP70, besides its chaperone function, plays an important role in the maintenance of the lysosome membrane stabilization. The results shown in Figure 4 indicate that both HSP70 and HSP90 were essential for PEL cell survival. Trypan blue exclusion was used in all the dose-response cytotoxic assay performed in this study.

To further explore how cell death occurred in PEL cells by the inhibition of the above-reported prosurvival pathways, we then performed a western-blot analysis of the poly (ADP-ribose) polymerase (PARP) cleavage. PARP cleavage generally represents a final event of an apoptotic cell death and is mainly mediated by caspase activation [51]. We found that, except for PES, previously shown to induce a necroptotic cell death type [40], all the other drugs utilized in this study induced the cleavage of PARP in PEL cells (Figure 5). Besides considering the cytotoxic effect, it is important to evaluate the side effect that anticancer drugs could have on the immune cells and DCs in particular, being cells with a pivotal role in the immune system [52]. Preliminary data obtained by exposing monocyte-derived DCs to all the drugs used against PEL cells, at the concentrations able to reduce $50 \%$ of PEL cell survival, showed that all of them, except for Bortezomib and Bayll-7082, were almost completely safe towards DCs (data not shown). 


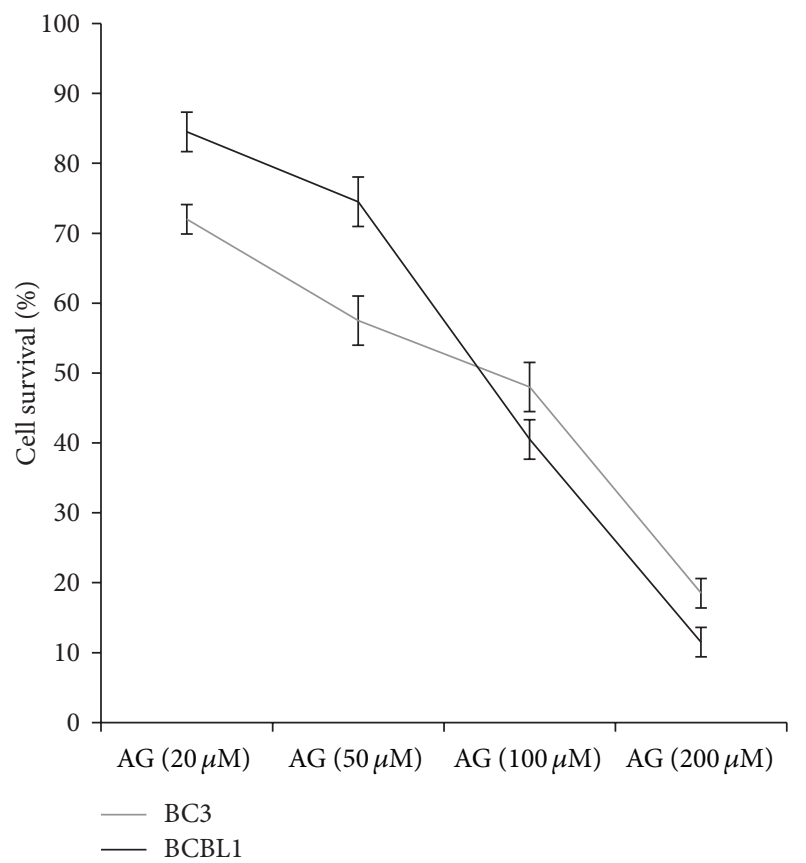

(a)

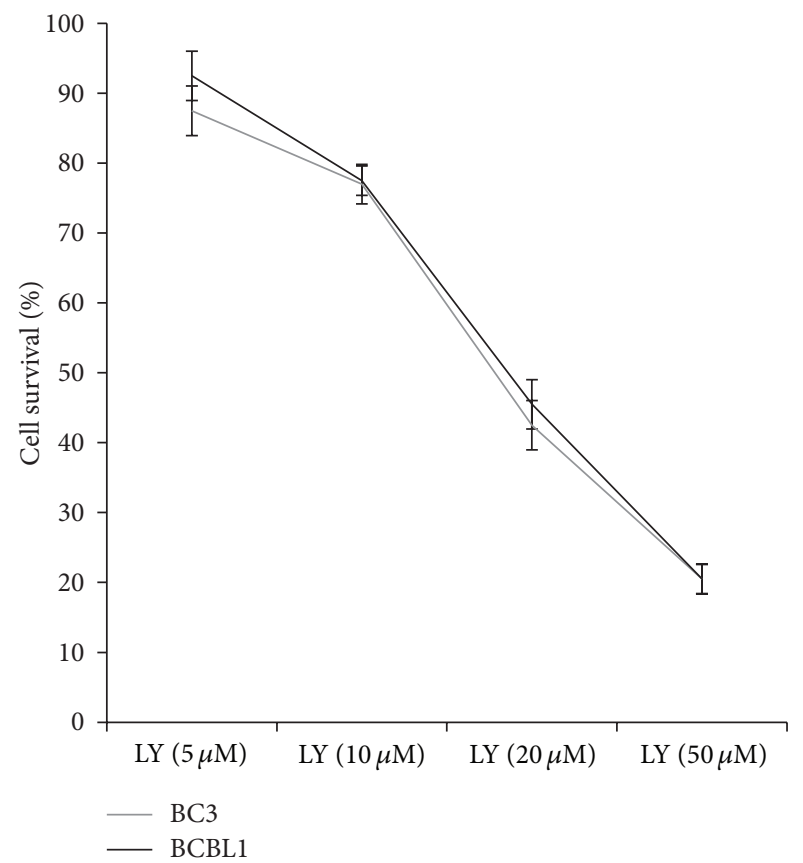

(b)

FIgURE 3: Dose-response cytotoxic assay of STAT3 or AKT inhibition in BC3 and BCBL1 PEL cells treated with AG490 (AG) (Calbiochem) or LY294002 (LY) (Santa Cruz), respectively, at the indicated doses. Trypan blue exclusion assays were performed after 24 hours of treatment. Mean \pm SD of three experiments is reported.

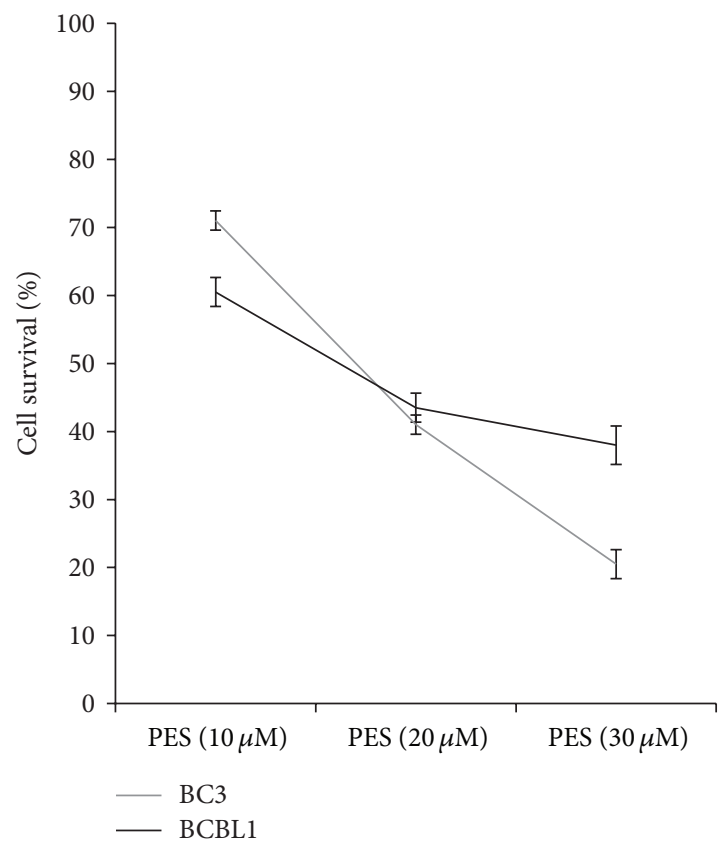

(a)

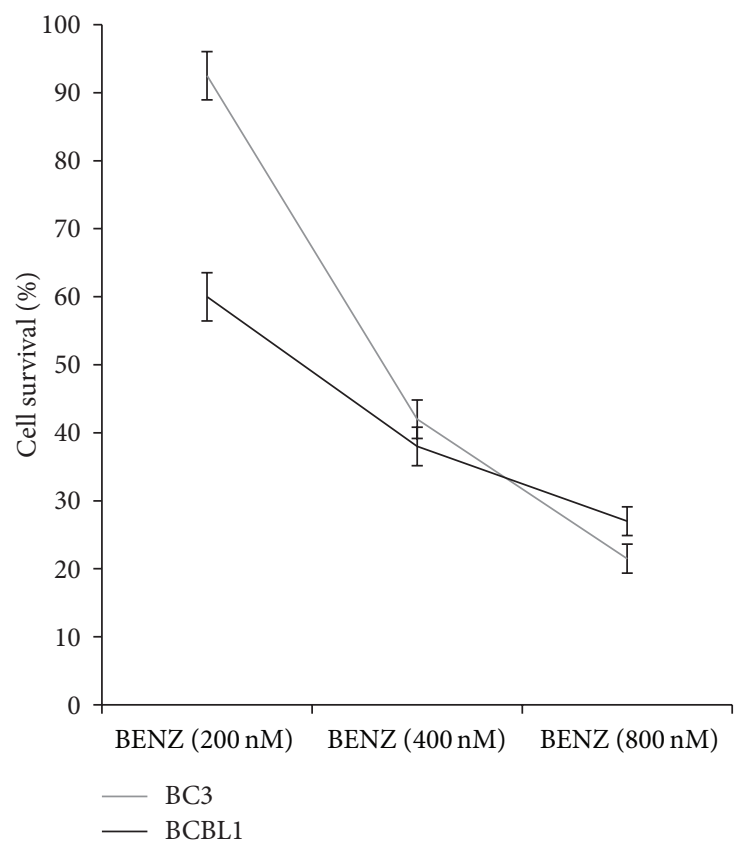

(b)

FIgURE 4: Dose-response cytotoxic assay of BC3 and BCBL1 PEL cells treated with PES (HSP70 inhibitor) (Calbiochem) and Benzisoxazole (BENZ) (HSP90 inhibitor) (Calbiochem), at the indicated doses. Trypan blue exclusion assays were performed after 24 hours of treatment and mean \pm SD of three independent experiments is reported. 


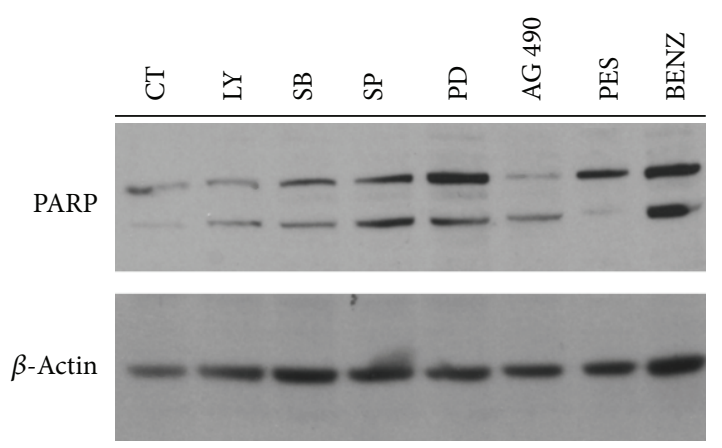

FIGURE 5: Western-blot analysis showing the PARP cleavage in BCBL1 cells treated for 24 hours with all the above reported cytotoxic drugs: LY294002 (LY), SB203580 (SB), SP600125 (SP), PD98059 (PD), AG490 (AG), PES, and Benzisoxazole (BENZ). $\beta$-Actin is included as loading control and a representative experiment out of three is reported. Antibodies against PARP (Cell Signaling) and $\beta$ actin (Sigma Aldrich) were diluted 1:1000 and 1:10000, respectively.

\section{Conclusion}

In conclusion, the use of drugs that target constitutively activated pathways in PEL cells could represent a valid alternative in translational therapies, especially considering the ability of most of them to induce an immunogenic cell death. In addition, their use is very promising because they have a low side effect towards the immune cells, and DCs in particular, rendering their use safer than conventional chemotherapies.

\section{Conflict of Interests}

The authors declare no conflict of interests.

\section{Acknowledgments}

This work was supported by MIUR, Associazione Italiana per la Ricerca (AIRC) no. 10265, and Pasteur Cenci-Bolognetti Foundation. The authors thank Sandro Valia, Marina Peddis, and Mariarosaria Filardi for technical assistance.

\section{References}

[1] C. Boshoff and R. Weiss, "AIDS-related malignancies," Nature Reviews Cancer, vol. 2, no. 5, pp. 373-382, 2002.

[2] R. G. Nador, E. Cesarman, A. Chadburn et al., "Primary effusion lymphoma: a distinct clinicopathologic entity associated with the Kaposi's sarcoma-associated herpes virus," Blood, vol. 88, no. 2, pp. 645-656, 1996.

[3] E. Cesarman, R. G. Nador, K. Aozasa, G. Delsol, J. W. Said, and D. M. Knowles, "Kaposi's sarcoma-associated herpesvirus in non-AIDS-related lymphomas occurring in body cavities," The American Journal of Pathology, vol. 149, no. 1, pp. 53-57, 1996.

[4] W. Fan, D. Bubman, A. Chadburn, W. J. Harrington Jr., E. Cesarman, and D. M. Knowles, "Distinct subsets of primary effusion lymphoma can be identified based on their cellular gene expression profile and viral association," Journal of Virology, vol. 79, no. 2, pp. 1244-1251, 2005.
[5] B. Ensoli and A. Cafaro, "Novel strategies toward the development of an effective vaccine to prevent human immunodeficiency virus infection or acquired immunodeficiency virus," AIDS Clinical Review, vol. 18, no. 4, pp. 23-61, 2000.

[6] H. G. Drexler, C. Meyer, G. Gaidano, and A. Carbone, "Constitutive cytokine production by primary effusion (body cavitybased) lymphoma-derived cell lines," Leukemia, vol. 13, no. 4, pp. 634-640, 1999.

[7] L. Haddad, H. El Hajj, R. Abou-Merhi et al., "KSHV-transformed primary effusion lymphoma cells induce a VEGFdependent angiogenesis and establish functional gap junctions with endothelial cells," Leukemia, vol. 22, no. 4, pp. 826-834, 2008.

[8] K. D. Jones, Y. Aoki, Y. Chang, P. S. Moore, R. Yarchoan, and G. Tosato, "Involvement of interleukin-10 (IL-10) and viral IL-6 in the spontaneous growth of Kaposi's sarcoma herpesvirusassociated infected primary effusion lymphoma cells," Blood, vol. 94, no. 8, pp. 2871-2879, 1999.

[9] J. Osborne, P. S. Moore, and Y. Chang, "KSHV-encoded viral IL- 6 activates multiple human IL-6 signaling pathways," Human Immunology, vol. 60, no. 10, pp. 921-927, 1999.

[10] R. Yamin, N. S. Kaynan, A. Glasner et al., "The viral KSHV chemokine vMIP-II inhibits the migration of naive and activated human NK cells by antagonizing two distinct chemokine receptors," PLoS Pathogens, vol. 9, no. 8, Article ID e1003568, 2013.

[11] G. Niu, K. L. Wright, M. Huang et al., "Constitutive stat3 activity up-regulates VEGF expression and tumor angiogenesis," Oncogene, vol. 21, no. 13, pp. 2000-2008, 2002.

[12] D. A. Braun, M. Fribourg, and S. C. Sealfon, "Cytokine response is determined by duration of receptor and signal transducers and activators of transcription 3 (STAT3) activation," The Journal of Biological Chemistry, vol. 288, no. 5, pp. 2986-2993, 2013.

[13] S. Uddin, A. R. Hussain, P. S. Manogaran et al., "Curcumin suppresses growth and induces apoptosis in primary effusion lymphoma," Oncogene, vol. 24, no. 47, pp. 7022-7030, 2005.

[14] Y. Aoki, G. M. Feldman, and G. Tosato, "Inhibition of STAT3 signaling induces apoptosis and decreases survivin expression in primary effusion lymphoma," Blood, vol. 101, no. 4, pp. 1535$1542,2003$.

[15] M. Cirone, L. Di Renzo, L. V. Lotti et al., "Primary effusion lymphoma cell death induced by bortezomib and AG 490 activates dendritic cells through CD91," PLoS ONE, vol. 7, no. 3, Article ID e31732, 2012.

[16] A. Garufi, G. Pistritto, C. Ceci et al., “Targeting COX-2/PGE pathway in HIPK2 knockdown cancer cells: impact on dendritic cell maturation," PLoS ONE, vol. 7, no. 11, Article ID e48342, 2012.

[17] Y. Ma, R. Conforti, L. Aymeric et al., "How to improve the immunogenicity of chemotherapy and radiotherapy," Cancer and Metastasis Reviews, vol. 30, no. 1, pp. 71-82, 2011.

[18] T. S. Griffith and T. A. Ferguson, "Cell death in the maintenance and abrogation of tolerance: the five Ws of dying cells," Immunity, vol. 35, no. 4, pp. 456-466, 2011.

[19] U. Bharadwaj, M. Li, R. Zhang, C. Chen, and Q. Yao, "Elevated interleukin-6 and G-CSF in human pancreatic cancer cell conditioned medium suppress dendritic cell differentiation and activation," Cancer Research, vol. 67, no. 11, pp. 5479-5488, 2007.

[20] H. Xu, Y. Zhang, Y. Hua, T. Chen, H. Wang, and W. Wu, "IL-12 p35 silenced dendritic cells modulate immune responses by 
blocking IL-12 signaling through JAK-STAT pathway in T lymphocytes," Biochemical and Biophysical Research Communications, vol. 353, no. 3, pp. 812-816, 2007.

[21] S. L. Fossey, M. D. Bear, W. C. Kisseberth, M. Pennell, and C. A. London, "Oncostatin M promotes STAT3 activation, VEGF production, and invasion in osteosarcoma cell lines," BMC Cancer, vol. 11, article 125, 2011.

[22] K. Arita, A. P. South, G. Hans-Filho et al., "Oncostatin M receptor- $\beta$ mutations underlie familial primary localized cutaneous amyloidosis," The American Journal of Human Genetics, vol. 82, no. 1, pp. 73-80, 2008.

[23] A. P. Bhatt and B. Damania, "AKTivation of PI3K/AKT/mTOR signaling pathway by KSHV," Frontiers in Immunology, vol. 3, Article ID Article 401, 2012.

[24] C. C. Tomlinson and B. Damania, "The K1 protein of Kaposi's Sarcoma-Associated Herpesvirus activates the AKT signaling pathway," Journal of Virology, vol. 78, no. 4, pp. 1918-1927, 2004.

[25] S. Uddin, A. R. Hussain, K. A. Al-Hussein et al., "Inhibition of phosphatidylinositol $3^{\prime}$-kinase/AKT signaling promotes apoptosis of primary effusion lymphoma cells," Clinical Cancer Research, vol. 11, no. 8, pp. 3102-3108, 2005.

[26] A. P. Bhatt, P. M. Bhende, S.-H. Sin, D. Roy, D. P. Dittmer, and B. Damania, "Dual inhibition of PI3K and mTOR inhibits autocrine and paracrine proliferative loops in PI3K/Akt/ mTOR-addicted lymphomas," Blood, vol. 115, no. 22, pp. 44554463, 2010.

[27] R. Gonnella, R. Santarelli, A. Farina et al., "Kaposi sarcoma associated herpesvirus (KSHV) induces AKT hyperphosphorylation, bortezomib-resistance and GLUT-1 plasma membrane exposure in THP-1 monocytic cell line," Journal of Experimental and Clinical Cancer Research, vol. 32, no. 1, article 79, 2013.

[28] S.-H. Sin, D. Roy, L. Wang et al., "Rapamycin is efficacious against primary effusion lymphoma (PEL) cell lines in vivo by inhibiting autocrine signaling," Blood, vol. 109, no. 5, pp. 21652173, 2007.

[29] G. L. Johnson and R. Lapadat, "Mitogen-activated protein kinase pathways mediated by ERK, JNK, and p38 protein kinases," Science, vol. 298, no. 5600, pp. 1911-1912, 2002.

[30] R. Dai, R. Chen, and H. Li, "Cross-talk between PI3K/Akt and MEK/ERK pathways mediates endoplasmic reticulum stressinduced cell cycle progression and cell death in human hepatocellular carcinoma cells," International Journal of Oncology, vol. 34, no. 6, pp. 1749-1757, 2009.

[31] M. Granato, R. Santarelli, L. V. Lotti et al., "JNK and macroautophagy activation by bortezomib has a pro-survival effect in primary effusion lymphoma cells," PLoS ONE, vol. 8, no. 9, Article ID e75965, 2013.

[32] M. Raciti, L. V. Lotti, S. Valia, F. M. Pulcinelli, and L. Di Renzo, "JNK2 is activated during ER stress and promotes cell survival," Cell Death and Disease, vol. 3, no. 11, article e429, 2012.

[33] S. A. Keller, E. J. Schattner, and E. Cesarman, "Inhibition of $\mathrm{NF}-\kappa \mathrm{B}$ induces apoptosis of KSHV-infected primary effusion lymphoma cells," Blood, vol. 96, no. 7, pp. 2537-2542, 2000.

[34] R. Gopalakrishnan, H. Matta, and P. M. Chaudhary, "A purine scaffold HSP90 inhibitor BIIB021 has selective activity against KSHV-associated primary effusion lymphoma and blocks vFLIP k13-induced NF- $\kappa$ B," Clinical Cancer Research, vol. 19, no. 18, pp. 5016-5026, 2013.

[35] H. Matta and P. M. Chaudhary, "The proteasome inhibitor Bortezomib (PS-341) inhibits growth and induces apoptosis in primary effusion lymphoma cells," Cancer Biology and Therapy, vol. 4, no. 1, pp. 77-82, 2005.
[36] P. G. Richardson, C. Mitsiades, T. Hideshima, and K. C. Anderson, "Bortezomib: proteasome inhibition as an effective anticancer therapy," Annual Review of Medicine, vol. 57, pp. 3347, 2006.

[37] M. Cirone, L. Di Renzo, L. V. Lotti et al., "Activation of dendritic cells by tumor cell death," OncoImmunology, vol. 1, no. 7, pp. 1218-1219, 2012.

[38] M. Chatterjee, S. Jain, T. Stühmer et al., "STAT3 and MAPK signaling maintain overexpression of heat shock proteins $90 \alpha$ and $\beta$ in multiple myeloma cells, which critically contribute to tumor-cell survival," Blood, vol. 109, no. 2, pp. 720-728, 2007.

[39] M. Chatterjee, M. Andrulis, T. Stühmer et al., "The PI3k/Akt signaling pathway regulates the expression of Hsp70, which critically contributes to Hsp90-chaperone function and tumor cell survival in multiple myeloma," Haematologica, vol. 98, no. 7, pp. 1132-1141, 2013.

[40] M. Granato, V. Lacconi, M. Peddis et al., "HSP70 inhibition by 2-phenylethynesulfonamide induces lysosomal cathepsin D release and immunogenic cell death in primary effusion lymphoma," Cell Death and Disease, vol. 4, no. 7, article e730, 2013.

[41] C. Higashi, C. Saji, K. Yamada et al., "The effects of heat shock protein 90 inhibitors on apoptosis and viral replication in primary effusion lymphoma cells," Biological and Pharmaceutical Bulletin, vol. 35, no. 5, pp. 725-730, 2012.

[42] U. Nayar, P. Lu, R. L. Goldstein et al., “Targeting the Hsp90-associated viral oncoproteome in gammaherpesvirus-associated malignancies," Blood, vol. 122, no. 16, pp. 2837-2847, 2013.

[43] B. Lamottke, M. Kaiser, M. Mieth et al., "The novel, orally bioavailable HSP90 inhibitor NVP-HSP990 induces cell cycle arrest and apoptosis in multiple myeloma cells and acts synergistically with melphalan by increased cleavage of caspases," European Journal of Haematology, vol. 88, no. 5, pp. 406-415, 2012.

[44] R. G. Jenner, K. Maillard, N. Cattini et al., "Kaposi’s sarcomaassociated herpesvirus-infected primary effusion lymphoma has a plasma cell gene expression profile," Proceedings of the National Academy of Sciences of the United States of America, vol. 100, no. 18, pp. 10399-10404, 2003.

[45] H.-J. Wen, Z. Yang, Y. Zhou, and C. Wood, "Enhancement of autophagy during lytic replication by the Kaposi's sarcomaassociated herpesvirus replication and transcription activator," Journal of Virology, vol. 84, no. 15, pp. 7448-7458, 2010.

[46] N. Kanarek and Y. Ben-Neriah, "Regulation of NF- $\kappa$ B by ubiquitination and degradation of the I $\kappa$ Bs," Immunological Reviews, vol. 246, no. 1, pp. 77-94, 2012.

[47] R. Santarelli, R. Gonnella, G. Di Giovenale et al., "STAT3 activation by KSHV correlates with IL-10, IL-6 and IL-23 release and an autophagic block in dendritic cells," Scientific Reports, vol. 4, article 4241, 2014.

[48] K. Wakao, T. Watanabe, T. Takadama et al., "Sangivamycin induces apoptosis by suppressing Erk signaling in primary effusion lymphoma cells," Biochemical and Biophysical Research Communications, vol. 444, no. 2, pp. 135-140, 2014.

[49] K. W. Wen and B. Damania, "Hsp90 and Hsp40/Erdj3 are required for the expression and anti-apoptotic function of KSHV K1," Oncogene, vol. 29, no. 24, pp. 3532-3544, 2010.

[50] W. Chen, S.-H. Sin, K. W. Wen, B. Damania, and D. P. Dittmer, "Hsp90 inhibitors are efficacious against Kaposi Sarcoma by enhancing the degradation of the essential viral gene LANA, of the viral co-receptor EphA2 as well as other client proteins," PLoS Pathogens, vol. 8, no. 11, Article ID e1003048, 2012. 
[51] C. Soldani and A. I. Scovassi, "Poly(ADP-ribose) polymerase-1 cleavage during apoptosis: an update," Apoptosis, vol. 7, no. 4, pp. 321-328, 2002.

[52] J. Banchereau, F. Briere, C. Caux et al., "Immunobiology of dendritic cells," Annual Review of Immunology, vol. 18, pp. 767811, 2000. 


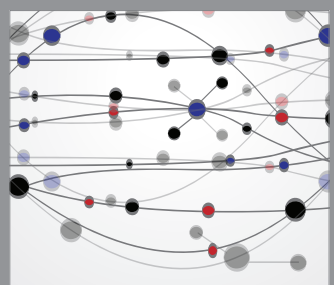

The Scientific World Journal
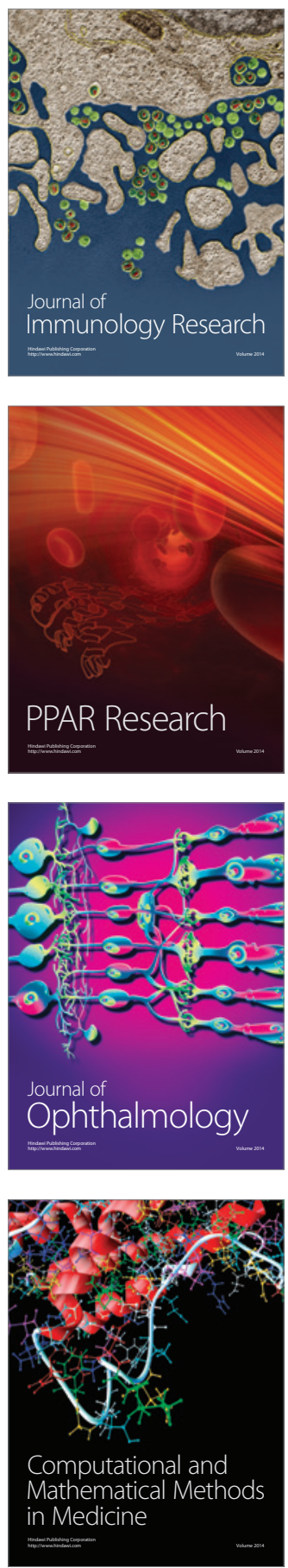

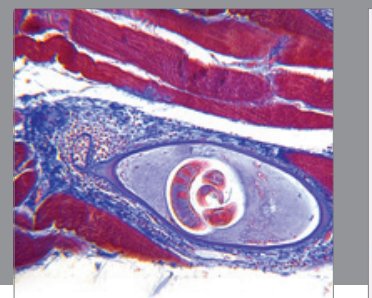

Gastroenterology

Research and Practice
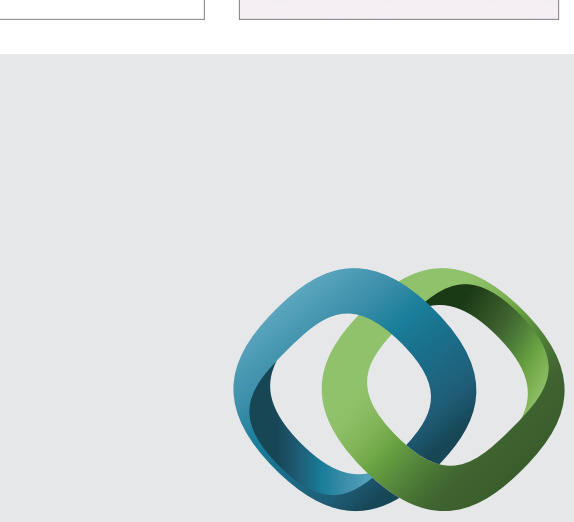

\section{Hindawi}

Submit your manuscripts at

http://www.hindawi.com
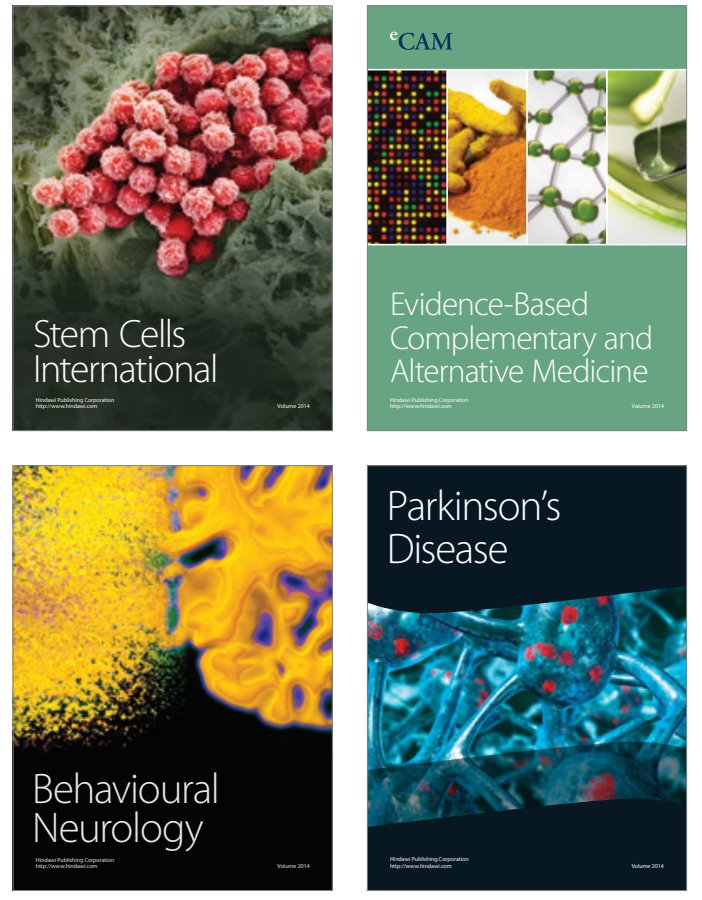
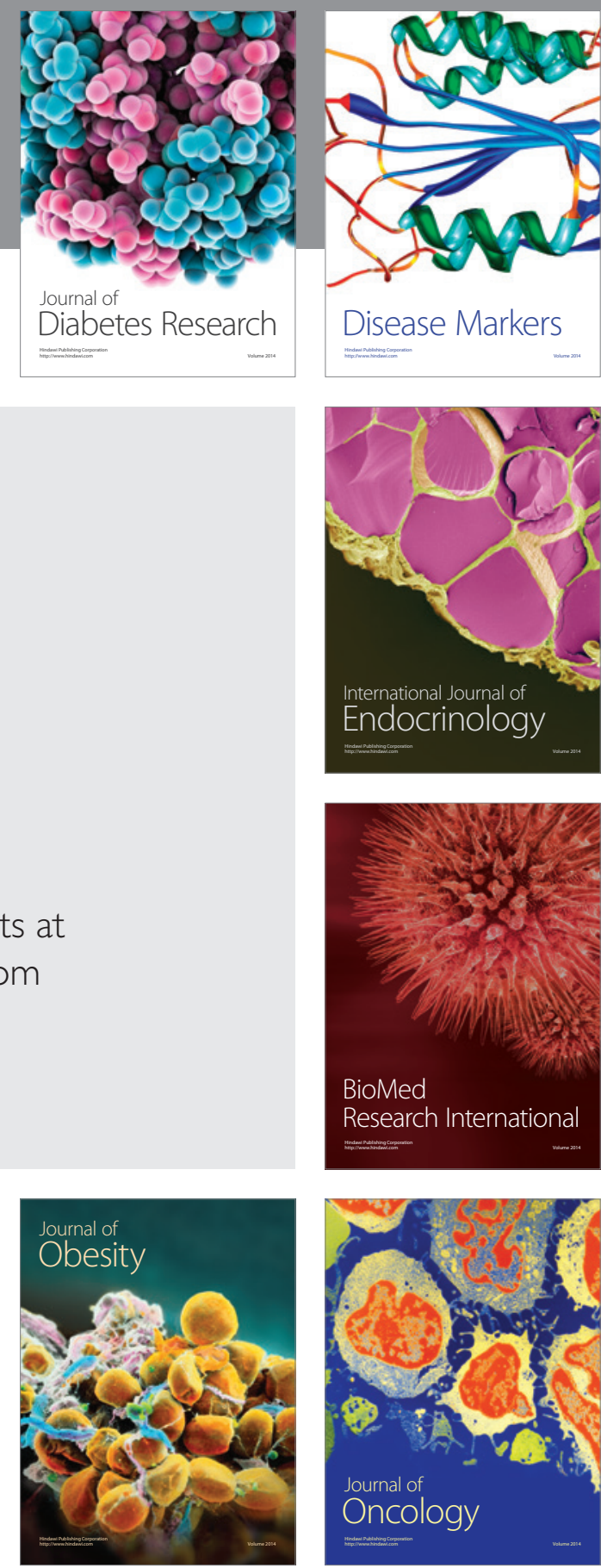

Disease Markers
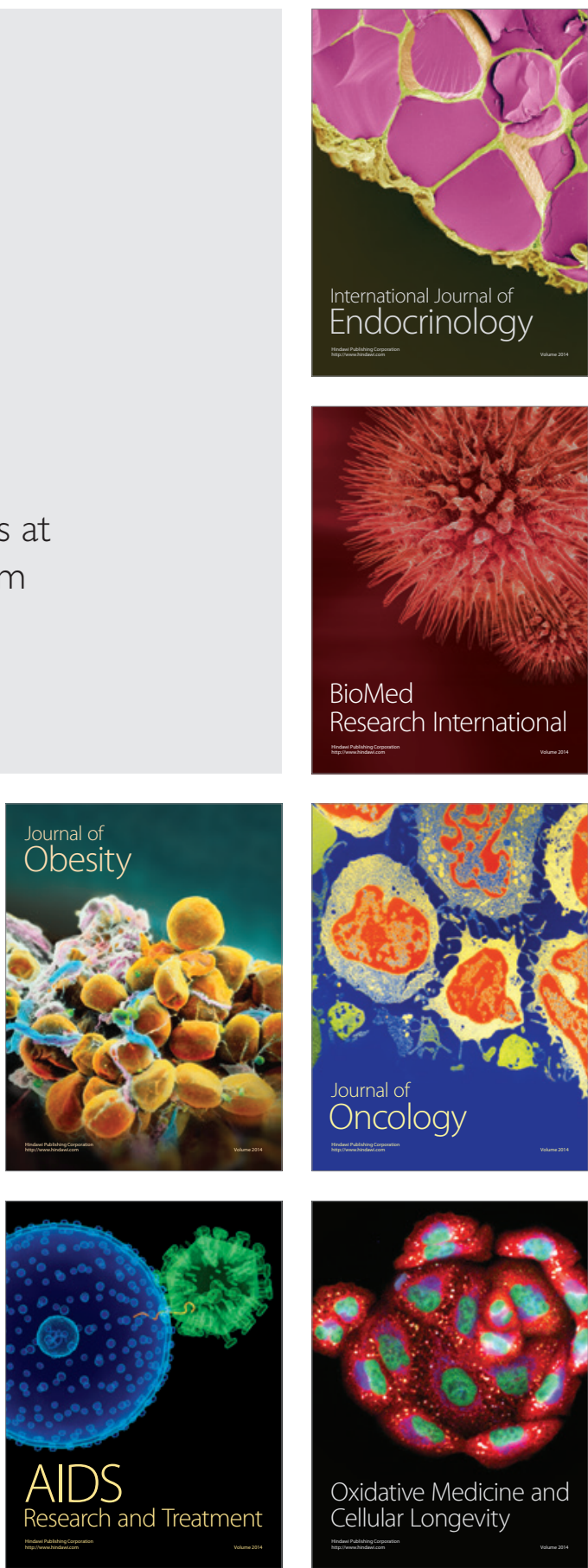